EGU2020-390

https://doi.org/10.5194/egusphere-egu2020-390

EGU General Assembly 2020

(c) Author(s) 2020. This work is distributed under

the Creative Commons Attribution 4.0 License.

\title{
High-resolution ensemble precipitation simulations over a small domain with complex topography
}

\author{
loannis Sofokleous ${ }^{1}$, Adriana Bruggeman ${ }^{1}$, Corrado Camera ${ }^{2}$, and George Zittis ${ }^{1}$ \\ ${ }^{1}$ The Cyprus Institute, Energy, Environment and Water Research Center, Nicosia, Cyprus (i.sofokleous@cyi.ac.cy) \\ ${ }^{2}$ Università degli Studi di Milano, Department of Earth Sciences, Milan, Italy
}

The reconstruction of detailed past weather and climate conditions, such as precipitation, is an essential part of hydrometeorological impact studies. Although this can be achieved through dynamical downscaling of reanalysis datasets, different model setup options can result in significantly different simulated fields. To select an efficient ensemble of the WRF atmospheric model for the simulation of precipitation at high resolution, suitable for hydrological studies at catchment scale, a series of simulation experiments is performed. The model experiments center on Cyprus, in the Eastern Mediterranean, a small domain with an area of $225 \times 145 \mathrm{~km}^{2}$ with complex topography. The simulations are made for the hydrologic year 2011-2012. Initial and boundary conditions are provided by the ERA 5 reanalysis dataset. A stepwise approach is followed for the evaluation of monthly simulations for an ensemble comprised of 18 combinations of various model physics parameterizations. In the first step, the model ensemble is evaluated for three domain setups with different extends and nested downscaling steps, i.e. $19 \cdot 10^{5} \mathrm{~km}^{2}$ with 12-, 4- and $1-\mathrm{km}$ grids (12-4-1), $19 \cdot 10^{5} \mathrm{~km}^{2}$ with 6 - and $1-\mathrm{km}$ grids $(6-1 \mathrm{a})$ and $7.28 \cdot 10^{5} \mathrm{~km}^{2}$ with 6 - and $1-\mathrm{km}$ grids (6-1b). The ensemble performance is then investigated for two initialization frequencies, 30 and 5 days, both with 6-hour spin-up. In the last step, the performance of the individual ensemble members is evaluated and the five best performing members are selected. A gridded precipitation dataset for the area over Cyprus is developed for the evaluation of the simulated precipitation. The statistical indicators used are bias, mean absolute error (MAE), NashSutcliffe efficiency and Kling-Gupta efficiency. The four indicators are scaled and combined in a single composite metric score (CMS), ranging from 0 to 1.

The best overall performance was achieved with the 12-4-1 domain setup. This setup resulted in the lowest bias of accumulated precipitation of the 18 -member ensemble, i.e. $1 \%$, compared to $8 \%$ for $6-1 \mathrm{a}$ and $10 \%$ for $6-1 \mathrm{~b}$, for the wet month of January. The 12-4-1 setup was also found to add value, in terms of computational time, to the least computationally demanding $6-1 b$ setup by reducing the monthly bias by $47 \mathrm{~mm}$ per $1000 \mathrm{cpu}$ hours. The statistical metrics for the ensemble with 5-day initialization exhibited very small variation from the metrics for the monthly initialization, with less than $4 \%$ difference in the MAE of the accumulated precipitation. The added value of the 5-day initialization, relative to the monthly initialization, was found to be negative for all four metrics in January and for two of the metrics in May. Despite the variable performance of individual ensemble members in different months, the combined metric showed that the overall 
highest (lowest) ranked members, with a CMS value of 0.63 (0.43), were those using the Ferrier and WRF-Double-Moment- $6^{\text {th }}$-class (WRF-Single-Moment- $6^{\text {th }}$-class) microphysical schemes. The proposed stepwise evaluation approach allows the identification of a reduced number of ensemble members, out of the initial ensemble, with a model setup that can simulate precipitation at high resolution and under different atmospheric conditions. 